Published in final edited form as:

IEEE Trans Biomed Eng. 2017 May ; 64(5): 1078-1088. doi:10.1109/TBME.2016.2588438.

\title{
Compartmental and Data-Based Modeling of Cerebral Hemodynamics: Nonlinear Analysis
}

\author{
B.C. Henley, \\ Department of Biomedical Engineering, University of Southern California, Los Angeles, CA 90089 \\ USA \\ D.C. Shin [IEEE Member], \\ Department of Biomedical Engineering, University of Southern California, Los Angeles, CA 90089 \\ USA
}

R. Zhang, and

Southwestern Medical Center, University of Texas

\section{V.Z. Marmarelis [IEEE Fellow]}

Department of Biomedical Engineering, University of Southern California, Los Angeles, CA 90089

USA

\section{Abstract}

Objective-As an extension to our study comparing a putative compartmental and data-based model of linear dynamic cerebral autoregulation $(\mathrm{CA})$ and $\mathrm{CO}_{2}$-vasomotor reactivity (VR), we study the CA-VR process in a nonlinear context.

Methods-We use the concept of Principal Dynamic Modes (PDM) in order to obtain a compact and more easily interpretable input-output model. This in silico study permits the use of input data with a dynamic range large enough to simulate the classic homeostatic CA and VR curves using a putative structural model of the regulatory control of the cerebral circulation. The PDM model obtained using theoretical and experimental data are compared.

Results-It was found that the PDM model was able to reflect accurately both the simulated static CA and VR curves in the Associated Nonlinear Functions (ANFs). Similar to experimental observations, the PDM model essentially separates the pressure-flow relationship into a linear component with fast dynamics and nonlinear components with slow dynamics. In addition, we found good qualitative agreement between the PDMs representing the dynamic theoretical and experimental $\mathrm{CO}_{2}$-flow relationship.

Conclusion-Under the modeling assumption and in light of other experimental findings, we hypothesize that PDMs obtained from experimental data correspond with passive fluid dynamical and active regulatory mechanisms.

Significance-Both hypothesis-based and data-based modeling approaches can be combined to offer some insight into the physiological basis of PDM model obtained from human experimental data. The PDM modeling approach potentially offers a practical way to quantify the status of specific regulatory mechanisms in the CA-VR process. 


\section{Index Terms}

Cerebral hemodynamics; parametric model; nonparametric model; principal dynamic modes

\section{Introduction}

Quantitative assessment of cerebral autoregulation (CA) and vasomotor reactivity (VR) in humans serves as an important modeling goal, due to the observed association between cerebrovascular structure and function in the outcome of head injury, stroke, vascular dementia, Alzheimer's disease, and other forms of cognitive impairment [2,4,5,8,13,22,29]. The availability of non-invasive measurements of relevant hemodynamic data has provided a means to obtain objective measures of CA and VR. It is generally accepted that the relationship between time variations in cerebral perfusion pressure (CPP), approximated by beat-to-beat measurements of mean arterial blood pressure (MABP), and cerebral blood flow velocity (CBFV) can reflect relevant information about the status of $\mathrm{CA}$ in humans $[2,3,9,10,15,23,33-38]$. In addition, it is well-known that $\mathrm{CO}_{2}$ tension, approximated by beat-to-beat measurements of end-tidal $\mathrm{CO}_{2}\left(\mathrm{ETCO}_{2}\right)$, significantly influences the cerebral vasculature under physiological conditions [2,11,17-19], and the relationship between variations in $\mathrm{ETCO}_{2}$ and $\mathrm{CBFV}$ is generally accepted as an approximation of the status of VR. Mathematical models of various forms have been employed as the methodological framework in which information about cerebral vascular status is summarized, analyzed, and interpreted [1,2,10-12,14,17,20,21,26-28,30-32,33-38]. These models can be classified generally as "hypothesis-based" and "data-based". In the hypothesis-based approach, parametric models are constructed in the form of differential and/or algebraic equations from first hemodynamic principles in attempt to explicate the observed data. The main benefit of these models is the ability to derive physiological meaning, since the parameters which characterize the model are given meaning a priori by the investigator. However, one important challenge is the virtually unavoidable oversimplification of the true depth of system, risking an incomplete and superficial picture of the actual system. This disadvantage can be supplemented by the use of inductive models, which are not hindered by simplifying assumptions about the system, but are instead obtained directly from measured data. Thus the data-based models are nonparametric in nature and the depth of the system model depends on the richness of the data. These models are typically much easier to obtain (using a computer), and knowledge about the system is not required. However, the challenge of the data-based approach lies in the task of clear physiological interpretation of the model, which says nothing explicitly about the system.

Since the main advantage of the hypothesis-based and data-based approaches supplement at least in part the disadvantage of each other, these two models have been used in the same modeling study in the pursuit of finding agreement between what is postulated and what is observed experimentally $[8,29]$. Achieving this goal would lead to a two-fold benefit: the postulated parametric model would be validated, and the nonparametric model would have a possible foundation to be interpreted. However, if there is a discrepancy between the two models, then improvements to the postulated model can be suggested, since the nonparametric model is viewed as an approximation of the true system. 
In the context of CA and VR, mathematical modeling studies are typically framed with the cerebral vasculature as the system of interest [19-21]. This system can be viewed in terms of the cerebral circulation (the vessels and their physical properties) and its interaction with regulatory mechanisms (myogenic, endothelial, neural, metabolic, etc.) that control the circulation in order to optimize cerebral perfusion to support brain health. The "response" of this system is viewed in terms of changes in cerebral blood flow (CBF) and the external "stimuli" of the system is viewed in terms of changes in $\mathrm{CPP}$ and $\mathrm{CO}_{2}$ tension. Thus the aim is to explain the causal effects of $\mathrm{CPP}$ and $\mathrm{CO}_{2}$ tension on $\mathrm{CBF}$ in terms of physical properties of the cerebral vasculature and its regulation. One putative hypothesis-based model has been proposed by Ursino, et al. [19], which employs the lumped-parameter modeling concept. In Ursino's cerebral hemodynamic $(\mathrm{CH})$ model, the cerebral circulation is represented by a series of hydraulic resistance and compliance elements, which represent respectively the tone and elasticity of the arterial and venous vasculature. The regulation of the cerebral vasculature is assumed to act through the alteration of arterial compliance and resistance. Specifically, CA is modeled as a flow-dependent feedback process that modifies compliance and resistance to counter-regulate the initial deviation in CBF. VR is modeled as a $\mathrm{CO}_{2}$ driven process that modifies compliance and resistance to increase $\mathrm{CBF}$ during hypercapnia and decrease CBF during hypocapnia. These two mechanisms are further assumed to interact through a nonlinear (sigmoidal) relationship, giving rise to the classical homeostatic relationship between $\mathrm{CPP}$ and $\mathrm{CBF}$ and sigmoidal static relationship between $\mathrm{CO}_{2}$ tension and $\mathrm{CBF}$.

The purpose of this study is to relate the postulated $\mathrm{CH}$ model with a nonparametric model that captures both the dynamic and static MABP-CBFV and $\mathrm{ETCO}_{2}-\mathrm{CBFV}$ relationship in a nonlinear context. To achieve this objective, we utilize the Principal Dynamic Mode (PDM) formulation of the Volterra model, which has been used in previous data-based studies of hemodynamic data [33-38]. The central question raised in this study is what is a PDM approximation of the $\mathrm{CH}$ model? The obtained PDM approximation is compared to experimental results using human hemodynamic data and the relation between the main features of the PDM model and key parameters in the $\mathrm{CH}$ model are explored.

\section{Modeling Methods}

\section{i. The Cerebral Hemodynamic (CH) Model}

The point of view of the hypothesis-based approach is that the main behavior exhibited in the CA-VR process can be emulated using differential and algebraic equations that characterize the manner in which the cerebral vascular system responds to changes in CPP and $\mathrm{CO}_{2}$ tension. As mentioned, a commonly used model is the compartmental model of the cerebral circulation $[6,10,13,19-21,28,31,32]$. In this approach, the cerebral vasculature is summarized by the interconnection of lumped parameters representing vascular resistance and compliance. The resistance describes the level of tone in the vessel and the compliance describes the ability of the vessel to distend in response to change in local transmural pressure. In this paper, we employ a cerebral hemodynamic $(\mathrm{CH})$ model, adopted from Ursino, et al [19] and used in other studies [6,31], since it is fairly compact and includes a description of the regulation of $\mathrm{CBF}$ during changes in $\mathrm{CPP}$ (autoregulation) and $\mathrm{CO}_{2}$ 
tension (reactivity). An analogy can be made between the $\mathrm{CH}$ model and an electrical circuit, where the hydraulic resistances can be seen as the opposition to electrical current (blood flow) and the compliances can be seen as the storage of charge (blood volume).

An equivalent circuit representation of the $\mathrm{CH}$ model is shown in Figure 1. The cerebral circulation is segmented into the cascade of arterial, capillary, and venous vascular compartments. The arterial segment is responsible for both the inflow of blood from the systemic circulation (not included) into the cranium and contributes to the regulation of blood flow during changes in $\mathrm{CPP}$ and $\mathrm{CO}_{2}$ tension. A common assumption here is that the large arteries, representing the internal carotid arteries leading up to the major cerebral arteries, are not significantly involved in the regulation of CBF through adjustment in vascular tone. The small arteries, representing the pial vessels on the surface of the brain and the parenchymal vessels penetrating the brain, are assumed as the main site of regulation through adjustments in vascular tone. As a result, the arterial segment is further segmented into "non-regulating" large arteries, characterized by the constant resistance $\mathrm{r}_{\mathrm{la}, 0}$, and "regulating" small arteries, characterized by the variable $r_{s a}(t)$.

A geometrical assumption is further imposed, representing the arterial compartment as a cylindrical tube with uniform length and inner radius, allowing for a simple relation between pressure, (uniform) flow, and resistance via Poiseuille's Law. Thus, resistance is inversely proportional the fourth power of the inner radius. Since the volume is proportional to the square of the inner radius, a relation between resistance and volume can be expressed:

$$
\mathrm{r}_{\mathrm{sa}}(\mathrm{t})=\mathrm{r}_{\mathrm{sa}, 0}\left[\frac{\mathrm{v}_{\mathrm{sa}, 0}}{\mathrm{v}_{\mathrm{sa}}(\mathrm{t})}\right]^{2}
$$

This implies that an increase and decrease in small arterial resistance, respectively mimicking vasoconstriction and dilation, occurs through the decrease and increase in volume. The volume of the non-regulating large arterial segment is held constant. The volume of the small arterial segment varies according to the compliance of the segment:

$$
\mathrm{v}_{\mathrm{sa}}(\mathrm{t})=\mathrm{c}_{\mathrm{a}}(\mathrm{t})\left[\mathrm{p}_{\mathrm{sa}}(\mathrm{t})-\mathrm{p}_{\mathrm{ic}, 0}\right]-\mathrm{v}_{\mathrm{la}, 0}
$$

and according to the conservation of mass (blood volume) principle:

$$
\frac{\mathrm{dv}_{\mathrm{sa}}}{\mathrm{dt}}=\mathrm{q}_{1 \mathrm{a}}(\mathrm{t})-\mathrm{q}_{\mathrm{sa}}(\mathrm{t})
$$

where flow through the large and small arterial segments are 


$$
\begin{gathered}
q_{1 a}(t)=\frac{p_{A}(t)-p_{s a}(t)}{r_{1 a, 0}+r_{s a}(t) / 2} \\
q_{s a}(t)=\frac{p_{s a}(t)-p_{v}(t)}{r_{s a}(t) / 2+r_{c v}, 0}
\end{gathered}
$$

The variables $\mathrm{P}_{\mathrm{a}}(\mathrm{t}), \mathrm{p}_{\mathrm{a}}(\mathrm{t})$, and $\mathrm{p}_{\mathrm{v}}(\mathrm{t})$ represent respectively the systemic, cerebral arterial, and cerebral venous pressure. The constant $\mathrm{p}_{\mathrm{ic}, 0}$ is intracranial pressure. Note that $\mathrm{p}_{\mathrm{A}}(\mathrm{t})$ is regarded as the signal representing MABP. It is observed in Eq 2 that an increase in compliance will cause an increase in volume, consequently a decrease in resistance, and thus vasodilation. Conversely, a decrease in compliance will cause a decrease in volume leading to an increase in resistance and vasoconstriction. As in [19], the small arterial resistance is bisected so that both the large and small arterial flow is influenced by alterations in resistance. For our purposes, it was found that this was necessary in order to simulate the static nonlinear MABP-CBFV relationship. We further adopt the assumption that a fraction of large arterial flow includes flow through the insonated middle cerebral artery (MCA). Thus, flow velocity, $\mathrm{f}_{\mathrm{v}}(\mathrm{t})$, can be computed as

$$
f_{v}(t)=c_{\text {mca }} \frac{q_{1 a}(t)}{A_{\text {mca }}}
$$

where $c_{m c a}=1 / 3$ and the cross sectional area $A_{m c a}=2 \pi \cdot(0.15 \mathrm{~cm})$ [19].

It is assumed that arterial compliance $\mathrm{c}_{\mathrm{a}}(\mathrm{t})$ is altered in response to changes in $\mathrm{CBF}$, mimicking $\mathrm{CA}$, and $\mathrm{CO}_{2}$ tension,

$$
\begin{gathered}
\mathrm{c}_{\mathrm{a}}(\mathrm{t})=\mathrm{c}_{\mathrm{a}, 0} \cdot\left(1+\frac{\Delta \mathrm{c}_{\mathrm{a}}}{2} \tanh \left[\frac{2 \times \mathrm{z}_{\mathrm{a}}(\mathrm{t})}{\Delta \mathrm{c}_{\mathrm{a}}}\right]\right) \\
\Delta \mathrm{c}_{\mathrm{a}}= \begin{cases}\mathrm{c}_{\mathrm{a}, \max } & \mathrm{z}_{\mathrm{a}}(\mathrm{t})>0 \\
\mathrm{c}_{\mathrm{a}, \min } & \mathrm{z}_{\mathrm{a}}(\mathrm{t}) \leq 0\end{cases} \\
\mathrm{z}_{\mathrm{a}}(\mathrm{t})=-\mathrm{g}_{\mathrm{q}} \cdot \mathrm{u}_{\mathrm{q}}(\mathrm{t})+\mathrm{g}_{\gamma} \cdot \mathrm{u}_{\gamma}(\mathrm{t})
\end{gathered}
$$




$$
\begin{aligned}
& \frac{\mathrm{du}}{\mathrm{dt}}=\frac{\mathrm{x}_{\mathrm{q}}(\mathrm{t})-\mathrm{u}_{\mathrm{q}}(\mathrm{t})}{\tau_{\mathrm{q}}} \\
& \mathrm{x}_{\mathrm{q}}(\mathrm{t})=\frac{\mathrm{q}_{\mathrm{sa}}(\mathrm{t})-\mathrm{q}_{0}}{\mathrm{q}_{0}} \\
& \frac{\mathrm{du}_{\gamma}}{\mathrm{dt}}=\frac{\mathrm{x}_{\gamma}(\mathrm{t})-\mathrm{u}_{\gamma}(\mathrm{t})}{\tau_{\gamma}} \\
& \mathrm{x}_{\gamma}(\mathrm{t})=\frac{\Delta \gamma}{2} \tanh \left\{\frac{2 \cdot\left[\frac{\gamma(\mathrm{t})-\gamma_{0}}{\gamma_{0}}\right]}{\Delta \gamma}\right\} \\
& \Delta \gamma= \begin{cases}\mathrm{x}_{\gamma, \max }, & {\left[\frac{\gamma(\mathrm{t})-\gamma_{0}}{\gamma_{0}}\right]>0} \\
\mathrm{x}_{\gamma, \min }, & {\left[\frac{\gamma(\mathrm{t})-\gamma_{0}}{\gamma_{0}}\right] \leq 0}\end{cases}
\end{aligned}
$$

In $\mathrm{Eq} 7$, the arterial compliance, $\mathrm{c}_{\mathrm{a}}(\mathrm{t})$, depends on the combined effects of autoregulation and reactivity, $\mathrm{z}_{\mathrm{a}}(\mathrm{t})(\mathrm{Eq} 8)$. The parameter $\Delta \mathrm{c}_{\mathrm{a}}$ is a term that is set to $\mathrm{c}_{\mathrm{a} \text {,max }}$ when $\mathrm{z}_{\mathrm{a}}(\mathrm{t})$ is positive (causing vasodilation) and $\mathrm{c}_{\mathrm{a} \text {, min }}$ when $\mathrm{z}_{\mathrm{a}}(\mathrm{t})$ is negative (causing vasoconstriction). This allows for the sigmoid to be generally asymmetric (when $c_{a, \max } \neq-\mathrm{c}_{\mathrm{a}, \min }$ ). The CA mechanism is defined by a flow-driven alteration in compliance, such that the resulting change in resistance opposes the initial change in flow (Eqs 9 \& 10). This effect is delayed, according to the simple first order filter in $\mathrm{Eq}$ 9, which is characterized by the time constant $\tau_{\mathrm{q}}$. The VR mechanism is defined by the alteration in compliance due to a change in $\mathrm{CO}_{2}$ tension (Eq 11), represented by the variable $\gamma(\mathrm{t})$, which is an exogenous variable with respect to the $\mathrm{CH}$ model (Eq 12). This effect is also defined by a simple first order filter in Eq 11, which is characterized by the time constant $\tau_{\gamma}$. In order to emulate the static relationship between $\mathrm{CBF}$ and $\mathrm{CO}_{2}$ tension, another asymmetric sigmoidal function used to introduce the total effect of the $\mathrm{CO}_{2}$ stimulus on compliance (Eqs 12). The parameter $\Delta \gamma$ is set to two values, $x_{\gamma, \max }$ or $x_{\gamma, \min }$, determining the maximum and minimum values of the stimulus for the VR mechanism. This in turn determines the threshold and saturation of the VR curve.

The resistance $r_{c v, 0}$ represents the capillary/venule resistance. The resistance $r_{l v, 0}$ and compliance $\mathrm{c}_{\mathrm{v} 0}$ of the venous compartment is assumed constant, and the predicted venous pressure $\mathrm{p}_{\mathrm{v}}(\mathrm{t})$ and volume $\mathrm{v}_{\mathrm{v}}(\mathrm{t})$ are defined as 


$$
\begin{gathered}
\mathrm{p}_{\mathrm{v}}(\mathrm{t})=\frac{\mathrm{v}_{\mathrm{v}}(\mathrm{t})}{\mathrm{c}_{\mathrm{v}, 0}}+\mathrm{p}_{\mathrm{ic}, 0} \\
\frac{\mathrm{dv}_{\mathrm{v}}}{\mathrm{dt}}=\mathrm{q}_{\mathrm{sa}}(\mathrm{t})-\frac{\mathrm{p}_{\mathrm{v}}(\mathrm{t})-\mathrm{p}_{\mathrm{V}, 0}}{\mathrm{r}_{\mathrm{lv}, 0}}
\end{gathered}
$$

where the constant $\mathrm{p}_{\mathrm{v}, 0}$ is the systemic venous pressure.

\section{ii. The Nonparametric Model}

As mentioned, it is assumed here that CPP can be approximated by the measured MABP, which is reasonable in light of the fact that systemic arterial pressure $(\approx 100 \mathrm{mmHg})$ is typically much greater than intracranial $(\approx 10 \mathrm{mmHg})$ or systemic venous pressure $(3-6$ $\mathrm{mmHg}$ ) [17,19-21,32]. In addition, $\mathrm{ETCO}_{2}$ measurements have been viewed as a reasonable (and non-invasive) predictor of arterial $\mathrm{CO}_{2}$ tension [33-38].

The transformation performed in the nonparametric approach is defined generally by the Volterra series, which can be expressed in the two-input case as shown in Eq 15.

The MABP input is represented by the variable $\mathrm{P}_{\mathrm{A}}(\mathrm{t}), \mathrm{ETCO}_{2}$ by the variable $\gamma(\mathrm{t})$, and CBFV by $f_{v}(t)$. The key modeling problem is to identify the unknown Volterra kernels, denoted by the letter " $k$ ", representing the time pattern of the effect of past and instantaneous variations in $\mathrm{MABP}, \mathrm{ETCO}_{2}$, and the interaction between the two inputs on the instantaneous value of CBFV. The two parameters, $\mathrm{M}_{\mathrm{p}}$ and $\mathrm{M}_{\gamma}$, define the memory extent for the MABP and $\mathrm{ETCO}_{2}$ inputs. In this paper, we rely on the Laguerre Expansion Technique (LET) to obtain an efficient estimate of the unknown kernels [39]. The Volterra kernels are expanded over a set of Laguerre basis functions which are assumed to span the functional space defined by the $\mathrm{CH}$ model. Further elaboration on the LET can be found in [38]. The key point here is that using the simulated input-output data of the $\mathrm{CH}$ model, the estimated kernels represent an approximation of the equivalent Volterra kernels of the $\mathrm{CH}$ model, which are assumed to exist over the dynamic range of the input signals.

The central concept of the nonparametric approach is the principal dynamic mode (PDM) formulation of the Volterra model. The PDM model is a compact form of the Volterra model that arises out of the pursuit to represent the model in terms of the smallest number of LTI filters (the PDMs) that can provide an adequate prediction of the model output, which is not generally achieved in the LET approach. A schematic of the PDM model is shown in Figure 2. The single dynamic nonlinear transformation in Eq 15 is essentially separated into two simpler transformations: a linear dynamic followed by a nonlinear static transformation. In this context, the input-output relationship in Eq 15 can be re-expressed in terms of the PDMs (Eq 19), where the functions $\left\{h_{\mathrm{jp}}(\tau)\right\}$ and $\left\{\mathrm{h}_{\mathrm{j} \gamma}(\tau)\right\}$ are respectively the PDMs of the MABP and $\mathrm{ETCO}_{2}$ input, while the functions $\left\{\mathrm{h}_{\mathrm{j}^{\prime} \mathrm{p}}(\tau)\right\}$ and $\left\{\mathrm{h}_{\mathrm{j}^{\prime}} \gamma(\tau)\right\}$ are the PDMs of the interaction between the two signals. The PDMs may be viewed as a "bank" or "resource" 
from which the dynamic nonlinear relationship between an input and the output is constructed.

To connect the PDM modeling approach to transfer function analysis (TFA), the PDMs are akin to the impulse response function (IRF) of the system input, in that they filter the input signal in the exact same way. However, they are not exactly the same, as the output of the IRF in TFA is the

$$
\begin{aligned}
\mathrm{f}_{\mathrm{v}}(\mathrm{t})= & \mathrm{f}_{\mathrm{v}, 0}+\sum_{\mathrm{qp}_{\mathrm{p}}=1}^{\mathrm{Q}_{\mathrm{p}}} \int_{0}^{\mathrm{M}_{\mathrm{p}}} \cdots \int_{0}^{\mathrm{M}_{\mathrm{p}}} \mathrm{k}_{\mathrm{qp}_{\mathrm{p}} \mathrm{p}}\left(\tau_{1, \mathrm{p}}, \ldots, \tau_{\mathrm{qp}_{\mathrm{p}}, \mathrm{p}}\right) \mathrm{p}_{\mathrm{A}}\left(\mathrm{t}-\tau_{1, \mathrm{p}}\right) \cdots \mathrm{p}_{\mathrm{A}}\left(\mathrm{t}-\tau_{\mathrm{q}_{\mathrm{p}}, \mathrm{p}}\right) \mathrm{d} \tau_{1, \mathrm{p}} \cdots \mathrm{d} \tau_{\mathrm{q}_{\mathrm{p}}, \mathrm{p}} \\
& +\sum_{\mathrm{q}_{\gamma}=1}^{\mathrm{Q}_{\mathrm{p}}} \int_{0}^{\mathrm{M}_{\gamma}} \cdots \int_{0}^{\mathrm{M}_{\gamma}} \mathrm{k}_{\mathrm{q}_{\gamma}, \gamma}\left(\tau_{1, \gamma}, \ldots, \tau_{\mathrm{q}_{\gamma}, \gamma}\right) \gamma\left(\mathrm{t}-\tau_{1, \gamma}\right) \cdots \gamma\left(\mathrm{t}-\tau_{\mathrm{q} \gamma, \gamma}\right) \mathrm{d} \tau_{1, \gamma} \cdots \mathrm{d} \tau_{\mathrm{q}_{\gamma}, \gamma} \\
& \quad+\int_{0}^{\mathrm{M}_{\mathrm{p}}} \int_{0}^{\mathrm{M}_{\gamma}} \mathrm{k}_{\mathrm{p}_{\gamma}, \gamma}\left(\tau_{\mathrm{p}}, \tau_{\gamma}\right) \mathrm{p}_{\mathrm{A}}\left(\mathrm{t}-\tau_{\mathrm{p}}\right) \gamma\left(\mathrm{t}-\tau_{\gamma}\right) \mathrm{d} \tau_{\gamma} \mathrm{d} \tau_{\mathrm{p}} \\
& +\int_{0}^{\mathrm{M}_{\mathrm{p}}} \int_{0}^{\mathrm{M}_{\mathrm{p}}} \int_{0}^{M_{\gamma}} \mathrm{k}_{\mathrm{p}, \mathrm{p}, \gamma}\left(\tau_{1, \mathrm{p}}, \tau_{2, \mathrm{p}}, \tau_{\gamma}\right) \mathrm{p}_{\mathrm{A}}\left(\mathrm{t}-\tau_{1, \mathrm{p}}\right) \mathrm{p}_{\mathrm{A}}\left(\mathrm{t}-\tau_{2, \mathrm{p}}\right) \gamma\left(\mathrm{t}-\tau_{\gamma}\right) \mathrm{d} \tau_{\gamma} \mathrm{d} \tau_{1, \mathrm{p}} \mathrm{d} \tau_{2, \mathrm{p}} \\
& +\int_{0}^{\mathrm{M}_{\mathrm{p}}} \int_{0}^{\mathrm{M}_{\gamma}} \int_{0}^{M_{\gamma}} \mathrm{k}_{\mathrm{p}, \gamma, \gamma}\left(\tau_{1, \mathrm{p}}, \tau_{1, \gamma}, \tau_{2, \gamma}\right) \mathrm{p}_{\mathrm{A}}\left(\mathrm{t}-\tau_{\mathrm{p}}\right) \gamma\left(\mathrm{t}-\tau_{1, \gamma}\right) \gamma\left(\mathrm{t}-\tau_{2, \gamma}\right) \mathrm{d} \tau_{1, \gamma} \mathrm{d} \tau_{2, \gamma} \mathrm{d} \tau_{\mathrm{p}}
\end{aligned}
$$

prediction of the system output, while the output of each PDM is a state variable of the system that is subsequently transformed statically by an associated nonlinear function (ANF) into an additive component of the system output. Therefore, the PDM model can be viewed as an extension to TFA by adding a layer of complexity, namely nonlinearity, to the analysis. The form of the ANFs can be viewed as a general polynomial expansion of the actual nonlinearity of the system.

The ANFs in the PDM model reflect the steady-state MABP-CBFV and $\mathrm{ETCO}_{2}-\mathrm{CBFV}$ relationship. As defined by the $\mathrm{CH}$ model, the steady-state MABP-CBFV relationship is determined by the steady-state change in pressure itself or a change in resistance. In the context of the $\mathrm{CH}$ model, the dependence on pressure alone is due to the classical Windkessel representation of the cerebral circulation, which predicts that flow will be proportional to pressure according to the current level of resistance and not its change. For this reason, this effect of pressure on flow can be referred to as "passive," and a rectilinear ANF is expected, where the coefficients corresponding to the higher degreed terms in the polynomial are sufficiently small enough to approximate the ANF as a scalar gain. However, if resistance is modulated, as is believed during regulatory action, the MABP-CBFV relationship will not be proportional, and an ANF with significant higher degree terms is expected. This effect is therefore considered "active." Finally, study of cerebral hemodynamics is often viewed in terms of pure oscillations in pressure and flow, giving rise to the concept of vascular impedance. In the frequency domain (expressed by the Fourier Transform of the PDMs), the ANFs can be used to infer the manner in which vascular impedance is modulated during oscillatory changes in the corresponding input signal. 


\section{i. Experimental Methods}

a. Synthetic Data Preparation-Ten sets of two 10 minute uniformly distributed signals were generated and low-pass filtered to represent "synthetic" beat-to-beat measurements of $\mathrm{MABP}$ and $\mathrm{ETCO}_{2}$. The $10 \mathrm{MABP}$ and $\mathrm{ETCO}_{2}$ signals were offset so that the mean values were respectively equal to the baseline values of $\mathrm{p}_{0, \mathrm{~A}}=100 \mathrm{mmHg}$ and $\gamma_{0}=40 \mathrm{mmHg}$. For the MABP input, values that were smaller than 40 or larger than $160 \mathrm{mmHg}$ were clipped at those values. Similarly, $\mathrm{ETCO}_{2}$ values that were smaller than 20 or larger than $60 \mathrm{mmHg}$ were clipped at those values.

b. Experimental Data Preparation-The highly sampled data of $\mathrm{MABP}, \mathrm{ETCO}_{2}$ and CBFV were reduced to beat-to-beat time-series data using averages over the respective R-R intervals, after removal of occasional measurement artifacts through application of hardclipping at $\pm 30 \%$ of average values. These beat-to-beat values were re-sampled every 0.25 seconds via cubic-spline interpolation and were high-pass filtered (via subtraction of a 2 minuite moving average using a Hanning window) to remove the constant baseline and very low frequency trends below $0.01 \mathrm{~Hz}$.

c. Nonparametric System Identification-In this paper, it is assumed that a Volterra series expansion of the $\mathrm{CH}$ model exists (Eqs 1-14), and that this expansion can be approximated adequately using LET up to at least the third order. The following steps are performed to obtain the LET and PDM model:

1. Using the parameters is Table 1, estimate the third order Volterra model defined in Eq 15 for each of the 10 synthetic input-output datasets separately.

2. For all 10 datasets, place the estimated first and second order kernels of the $\mathrm{MABP}$ and $\mathrm{ETCO}_{2}$ inputs into the columns of two respective rectangular matrices, with each second order kernel respectively scaled by the standard deviations of its corresponding inputs.

3. Apply singular value decomposition (SVD) on the matrices individually. Select the singular vectors for both inputs, $\left\{\mathrm{h}_{1, \mathrm{p}}, \mathrm{h}_{2, \mathrm{p}}, \mathrm{h}_{\mathrm{H}, \mathrm{p}}\right\}$ and $\left\{\mathrm{h}_{1, \gamma}, \mathrm{h}_{2, \gamma}, \mathrm{h}_{\mathrm{H}, \gamma}\right\}$. These singular vectors are the PDMs of the MABP and $\mathrm{ETCO}_{2}$ inputs, respectively.

4. For all 10 datasets, place the second order cross kernels of the MABP and $\mathrm{ETCO}_{2}$ input into the columns of a rectangular matrix.

5. Apply SVD on the cross kernel matrix. Select the desired vectors $\left\{h^{\prime}{ }_{p}\right\}$ for the MABP input. Repeat on the transpose of the cross kernel matrix to obtain the desired vectors $\left\{h^{\prime}{ }_{\gamma}\right\}$ for the $\mathrm{ETCO}_{2}$ input.

6. Perform steps 1-5 with the experimental input-output data from the 10 randomly selected healthy human subjects to obtain the experimental global PDMs.

d. Static and Dynamic Simulations-In order to assess the performance of the nonparametric approach in capturing the main static and dynamic behavior exhibited in the $\mathrm{CH}$ model, both the $\mathrm{CH}$ model and nonparametric model are exercised with step signals of various amplitudes and the predicted response of both are compared. The main question is 
whether the PDM model can capture accurately both the static and dynamic MABP- and $\mathrm{ETCO}_{2}$ - driven response under various conditions as predicted by the $\mathrm{CH}$ model.

\section{Modeling Results}

The six steps described in the Experimental Methods are performed to collect the data. The first important issue to address is the LET model structure. This is determined by the kernel orders $Q_{p}$ and $Q_{\gamma}$, number of Laguerres $L_{p}$ and $L_{\gamma}$, and the Laguerre parameters $a_{p}$ and $a_{\gamma}$. Since our target system is known (the $\mathrm{CH}$ model), we can use its structure to infer an appropriate nonparametric model structure. The static CA and VR curves both exhibit an inflection point, where the rate of change in the slope of the curves change signs.

Thus, a cubic (third order) kernel order is minimally required. It was found that accurate prediction of the CA and VR curves simultaneously can only be achieved when $Q_{p}=Q_{\gamma}=3$ (not shown). Since higher order kernels are computationally burdensome, we limit the kernel orders to cubic. The two remaining parameters, $L$ and $a$, specify the Laguerre basis used for kernel expansion and determine respectively the shape and time extent (number of significant values) of the dynamic relationship between the inputs and output. For optimal estimation, we desire the fewest number of basis functions (minimum $L_{p}$ and $L_{\gamma}$ ) and best values of $a_{p}$ and $a_{\gamma}$ that approximate adequately the static and dynamic CA-VR system simulated in the $\mathrm{CH}$ model. $L_{p}$ and $L_{\gamma}$ were adjusted manually while fixing $a_{p}=0.5$ and $a_{\gamma}$ $=0.85$, and it was found that $L_{p}=4$ and $L_{\gamma}=3$ provided good prediction (not shown). The values for $a_{p}$ and $a_{\gamma}$ were selected to best reflect the dynamic response predicted in the $\mathrm{CH}$ model.

The LET model is validated by computing normalized mean squared error (NMSE) of the model output prediction. The output NMSE, averaged between the 10 simulations, was 7.34 $\pm 4.66 \%$, indicating good predictive performance. As a result, we infer that the estimated Volterra kernels yield an accurate approximation of the equivalent kernels of the $\mathrm{CH}$ model. Following the procedure outlined in Experimental Methods, the PDMs of the MABP and $\mathrm{ETCO}_{2}$ input, $\left\{h_{p}\right\}$ and $\left\{h_{\gamma}\right\}$, were computed separately via SVD of the rectangular matrix containing their 10 respective first and second order kernels. In addition, the cross PDMs of the interaction between the two inputs, $\left\{h_{p}^{\prime}\right\}$ and $\left\{h^{\prime}{ }_{\gamma}\right\}$, were computed via SVD of the rectangular matrix containing the 10 estimated cross-kernels. The goal is to find the smallest number of LTI filters that can capture adequately the main dynamic and static behavior of the $\mathrm{CH}$ model. It was found that three of the PDMs for the MABP input $\left(H_{p}=3\right)$, three of the PDMs for the $\mathrm{ETCO}_{2}$ input $\left(H_{\gamma}=3\right)$, and one cross PDM for each input $\left(H_{p}^{\prime}=H_{\gamma}^{\prime}=1\right)$ were adequate to achieve this end (Table 1). The average NMSE of the PDM model output prediction between the 10 simulations was $15.16 \pm 1.22 \%$. in the noise-free case. This reduction in model performance (about $8.00 \%$ ) should be considered in light of the significant reduction in the number of free parameters between the LET (120) and PDM model (20).

To confirm that the PDM model structure was able to capture adequately the static behavior of the $\mathrm{CH}$ model, step signals of various amplitudes were applied at the MABP and $\mathrm{ETCO}_{2}$ inputs and the resulting response in $\mathrm{CBFV}$ predicted by the $\mathrm{CH}$ model was recorded. The 
CA curve predicted by the $\mathrm{CH}$ model can be reconstructed simply by exercising the $\mathrm{CH}$ model with step signals at the MABP input with amplitudes ranging over 40 to $160 \mathrm{mmHg}$ while the $\mathrm{ETCO}_{2}$ input is "clamped" at its baseline value $\left(\gamma_{0}-40 \mathrm{mmHg}\right.$. Similarly, the VR curve predicted by the $\mathrm{CH}$ model can be reconstructed simply by exercising the $\mathrm{CH}$ model with step signals at the $\mathrm{ETCO}_{2}$ input with amplitudes ranging over 20 to $60 \mathrm{mmHg}$ while the MABP input is "clamped" at its baseline value $\left(p_{0, A}=100 \mathrm{mmHg}\right)$. Since the dynamic range of the simulated input data cover these ranges, the obtained nonparametric model can also be exercised with the same signals. The steady state response in CBFV predicted by the CH, LET, and PDM models are shown in Figure 3. It is evident that both the LET and PDM model yield a relatively good prediction of the CA curve, with only small reduction in the PDM model predictions (left panel). The asymmetric sigmoidal VR curve appears to be captured by the LET and PDM models (right panel), however, the estimated central slope (between 30 and $50 \mathrm{mmHg}$ ) is slightly under-estimated and the PDM model over-estimates the VR curve more than the LET model for values of $\mathrm{ETCO}_{2}$ below 30 $\mathrm{mmHg}$.

In the PDM modeling approach, the MABP-CBFV relationship is found to be characterized by three branches (Figure 4, bottom row and Figure 5, right). The PDM in the first branch exhibits a high-pass characteristic, with an ANF that is mostly linear. In the time domain, a relatively large value at zero-lag is observed, followed by an undershoot that lasts for a couple seconds. PDM \#2 and \#3 in the second and third branch exhibit a slower dynamic than the first, with a resonant peak near 0.22 and $0.10 \mathrm{~Hz}$, respectively.

Both ANFs are nonlinear, with the ANF in the third branch exhibiting a strong cubic nonlinearity. In Figure 4, the theoretical PDMs (bottom row) are compared to the those obtained from experimental input-output data (top row). It is observed that the experimental PDMs show fairly close agreement with the theoretical PDMs. Since the mean dynamic range of the experimental MABP measurements (-8.63 to $9.12 \mathrm{mmHg}$ ) is much smaller than the synthetic data ( -60 to $60 \mathrm{mmHg}$ ), the ANFs estimated from the experimental data will be much smaller in magnitude. However, we note that both the theoretical and experimental ANF of the first branch is mostly linear, while the ANF of the second and third PDM are nonlinear.

Figures 6 and 7 displays the computed PDMs and estimated ANFs of the $\mathrm{ETCO}_{2}$ input. Like the MABP input, the mean dynamic range of the experimental $\mathrm{ETCO}_{2}$ measurements $(-3.55$ to $2.57 \mathrm{mmHg}$ ) is much smaller than the synthetic data, which had to be extended (-20 to 60 $\mathrm{mmHg}$ ) in order to observe the nonlinear effects predicted by the $\mathrm{CH}$ model. As a result, we point out that the first branch dominates the $\mathrm{ETCO}_{2}-\mathrm{CBFV}$ relationship. PDM \#1 is mainly integrative, and exhibits a small second positive phase. The corresponding ANF exhibits an asymmetric sigmoidal nonlinearity, with the threshold end of the sigmoid being smaller in magnitude than the saturating end. The second and third branches offer a much smaller contribution to the output. As with the MABP input, there is a strikingly good agreement between the experimental and theoretical PDMs, given the relative simplicity of the $\mathrm{CH}$ model (Figure 1). However, we note that the ANFs averaged between the 10 experimental datasets are very different from ANFs obtained from the synthetic datasets (Figures 7). 
Following Steps 4 and 5 of the Nonparametric System Identification procedure, we obtain the PDMs of the interaction between the MABP and $\mathrm{ETCO}_{2}$ inputs, quantifying the MABP$\mathrm{ETCO}_{2}$-CBFV relationship. Due to the constraints of space, the PDMs are not shown. However, we report that one PDM for each input is adequate to capture the second order cross kernel. The PDM for the MABP input exhibits a high-pass characteristic, similar to the first order kernel and first PDM of the MABP-CBFV relationship. The PDM for the $\mathrm{ETCO}_{2}$ input is mainly integrative and is similar to the first order kernel and first PDM of the $\mathrm{ETCO}_{2}$-CBFV relationship.

\section{Discussion}

The purpose of this study is to compare a parametric with a nonparametric nonlinear model of the CA-VR process. This is intended as an extension to a previous comparison study, which was constrained to a linear context [6]. In the latter study, a linear approximation of the $\mathrm{CH}$ model was derived analytically for the IRFs of the MABP and $\mathrm{ETCO}_{2}$ inputs and compared to experimental IRFs obtained from the input-output data of eight human subjects. The experimental data were obtained under resting conditions and, therefore, stayed well within the homeostatic plateau of the pressure-flow relation that can be approximated well by a linear model. The extension of experimental data outside the homeostatic plateau would require strenuous external pressure forcing imposed on the subject and was not feasible or allowable under the IRB approved protocol. Therefore, the study of the nonlinearities of the CA-VR process is feasible only through large-amplitude simulations of the $\mathrm{CH}$ model, and direct comparison with experimental findings is not possible under the present constraints. Therefore, the nonlinear modeling study of the CA-VR process is performed in this paper in silico by simulating the parametric $\mathrm{CH}$ model with large amplitude $\mathrm{MABP}$ and $\mathrm{ETCO}_{2}$ inputs and analyzing the resulting data in the context of nonparametric modeling. The largeamplitude nonparametric results are also compared with the experimental (restingamplitude) results. The goal of the study is two-fold: (1) to examine whether nonparametric models can capture the essential nonlinearities of this system under large-amplitude forcing conditions, and (2) to examine the resulting form of the nonparametric model under these conditions.

The experimental studies are limited in their ability to capture the strong system nonlinearities, because the dynamic range of the experimental inputs cannot exceed what is considered safe for the subject. Of course, this limitation does not exist for the simulation study, where sufficiently large amplitude range can be used for the MABP and $\mathrm{ETCO}_{2}$ inputs in order to capture the homeostatic nonlinearity of the $\mathrm{CH}$ model. However, it is not appropriate to compare the "large-amplitude" estimates of the system nonlinearity with the "small-amplitude" estimates derived from the experimental data, because the employed estimation method minimizes the mean-square error within the dynamic range of the data and may yield different estimates for different dynamic ranges (due to the employed cubic approximation). Therefore, it is difficult to determine whether a nonlinear model obtained from the analysis of spontaneous data under resting conditions can be "extrapolated" to larger dynamic ranges. We take the view that this "extrapolation" is not appropriate in the present context, because it engenders risks of misleading conclusions. Nonetheless, the PDM-based nonlinear modeling within the experimental data range yields better model 
prediction and useful information consistent with our current understanding of the mechanisms in the CA and VR process. This is corroborated by the surprising qualitative agreement between the obtained theoretical and experimental PDMs.

It was observed that the first branch in the MABP-CBFV relationship exhibited the traditional high-pass dynamic characteristic that is consistently observed in TFA $[1,3,13,24,25,29]$. The corresponding ANF is mainly rectilinear and was shown to dominate the linear dynamic MABP-CBFV relationship. The second and third branches both exhibited a dynamic characteristic inconsistent with the traditional high-pass characteristic, with PDM \#2 exhibiting a resonant peak around $0.22 \mathrm{~Hz}$ and PDM \#3 having a peak at about $0.10 \mathrm{~Hz}$. The corresponding ANFs of the second and third branch were more nonlinear than the first branch, with the third branch containing a strong cubic ANF. Similar to the MABP input, three branches were used to capture the main static and dynamic $\mathrm{ETCO}_{2}-\mathrm{CBFV}$ relationship. The first theoretical PDM of the $\mathrm{ETCO}_{2}$ was integrative with a small second positive phase, and the corresponding ANF exhibited a dominant asymmetric sigmoidal nonlinearity. The second and third branch exhibited slightly more complex behavior with their corresponding ANFs much weaker than the first.

Upon inspection of Figures 4 and 6 that present the obtained PDMs for large-amplitude simulated data and resting-amplitude experimental data, we observe surprisingly good agreement between the two sets of PDMs. However, this agreement does not hold for the obtained ANFs, especially for the $\mathrm{ETCO}_{2}$ input (Figures 5 and 7). It was found that the CA curve could be adequately captured by the first three PDMs of MABP-CBFV relationship (Figure 3, left). Both the experimental and theoretical models exhibit a fast-linear and two slow-nonlinear branches for the MABP input. One possible interpretation of this result is that the PDM model essentially separates the MABP-CBFV relationship into fast and slow dynamics, where the fast mode is mainly "passive," explaining dynamic effects not influenced by active changes in resistance, and the slower modes are mainly "active," explaining the dynamic effects influenced by active alterations in resistance caused by regulatory activity of (possibly) the autonomic nervous system. This apparent separation of linear and nonlinear effects is intriguing in that it may provide a connection between PDM analysis and the widely used linear TFA $[1,3,6,14,25,27,31,33]$. That is, if one desired to limit their study to linear analysis, one could simply take the first branch of the MABPCBFV as an approximation of the actual model, yielding a mainly linear model exhibiting a high-pass characteristic similar to TFA. This suggests that the PDM modeling approach can be viewed as a generalization of TFA. Similar to the MABP input, it was found that three PDMs for the $\mathrm{ETCO}_{2}$ input may be used to capture adequately the VR curve. These three PDMs also were similar for the simulated and experiential data, a striking result, given the relative simplicity of the $\mathrm{CH}$ model.

The main assumptions discussed in the previously published linear analysis [6] also apply here, besides the constraint of linearity. We note again that the CA and VR processes defined in the $\mathrm{CH}$ model both assume the form of a single first-order differential equation between changes in $\mathrm{CBF}$ and arterial compliance. While the qualitative similarities between the theoretical and experimental PDMs are striking, it is clear that the $\mathrm{CH}$ model can be improved by extending the single compartment assumption of CA and VR, due to the 
observation that the experimental PDM model is characterized by at least three PDMs for both inputs [33-36]. One consideration in modifying the $\mathrm{CH}$ model is to replace the single compartment with the interaction of multiple compartments, and observe whether the PDM model extracts each mechanism in individual branches. Another important limitation of this study was the non-physiological range of simulation data that was used in order to capture the nonlinearity in the $\mathrm{CH}$ model. The dynamic range of simulated $\mathrm{MABP}$ and $\mathrm{ETCO}_{2}$ data had to be increased to cover respectively the ranges of 40 to $160 \mathrm{mmHg}$ and 20 to $60 \mathrm{mmHg}$. However, the surprising qualitative agreement between the theoretical and experimental PDMs serves to illustrate the potential utility of the PDM modeling approach, as well as provide another quantitative way to explore more complex models of cerebral vascular function.

\section{Conclusion}

Under the stated modeling assumptions, the physiological interpretation of the obtained PDMs allows the formulation of potentially useful hypotheses regarding the specific mechanisms serving the processes of cerebral autoregulation and vasoreactivity. For example, it was found through the analysis of simulated data from the $\mathrm{CH}$ model that its third MABP-PDM is responsible for the nonlinear segments of the steady-state pressureflow curve outside the homeostatic plateau. When this modeling finding is combined with the experimental finding reported in [16] that these nonlinear segments were attributable to the myogenic mechanism, we can infer that the third MABP-PDM corresponds to the myogenic mechanism [39]. Following similar comparisons with experimental observations reported in the literature, we may posit that the first PDM of the MABP input corresponds mainly to the passive fluid dynamic (Windkessel) properties of the cerebral circulation, since it exhibits a high-pass characteristic above approximately $0.1 \mathrm{~Hz}$, with a small contribution from the sympathetic mechanism, being active primarily in frequencies below $0.1 \mathrm{~Hz}$, since the elimination of the $\mathrm{CA}$ gain in the $\mathrm{CH}$ model results in increased gain over these frequencies. Finally, the second MABP-PDM may be related to the cholinergic mechanism, since it makes a small positive linear contribution to the steady-state pressure-flow curve, consistent with the experimental observations reported in [16]. The connection between the obtained PDMs and physiological mechanisms must be explored further in the future, because it can advance significantly our scientific understanding of cerebral autoregulation and allow useful clinical applications.

\section{Acknowledgments}

This work was supported by the National Institutes of Health under Grant P41 EB-001978 through the Biomedical Simulations Resource at the University of Southern California. The authors wish to thank Professor John Clark for helpful suggestions in the initial stages of this study.

The work in this paper was supported in part by NIH grant P41 EB-001978 to the Biomedical Simulations Resource at USC.

\section{References}

1. Meel-van den Abeelen AS, et al. Transfer function analysis for the assessment of cerebral autoregulation using spontaneous oscillations in blood pressure and cerebral blood flow. Med Eng Phys. 2014; 36(5):563-575. [PubMed: 24721458] 
2. Meel-van den Abeelen AS, et al. Impaired cerebral autoregulation and vasomotor reactivity in sporadic Alzheimer's disease. Curr Alz Res. 11:11-7.

3. Blaber AP, et al. Transfer function analysis of cerebral autoregulation dynamics in autonomic failure patients. Stroke. 1997; 28(9):1686-1692. [PubMed: 9303010]

4. van Beck AH, et al. Cerebral autoregulation: overview of current concepts and methodology with special focus on the elderly. J Cereb Blood Flow Metab. 2008; 28:1071-1085. [PubMed: 18349877]

5. Viswanathan A, Greenberg SM. Cerebral Amyloid Angiopathy in the Elderly. Ann Neurol. 2011; 79:871-80.

6. Henley BC, et al. Compartmental and data-based modeling of cerebral hemodynamics: Linear Analysis. IEEE Access. 2015; 3:2317-2332. [PubMed: 26900535]

7. Lathi, BP. Linear Systems and Signals. Second. Oxford Univ Press; 2005. p. 371-72.

8. Iadecola C. Neurovascular regulation in the normal brain and in Alzheimer's disease. Nat Rev Neurosci. 2004; 5:347-360. [PubMed: 15100718]

9. Gommer ED, et al. Dynamic cerebral autoregulation in subjects with Alzheimer's disease, mild cognitive impairment, and controls: evidence for increased peripheral vascular resistance with possible predictive value. J Alz Dis. 2012; 30(4):805-813.

10. Tiecks FP, et al. Comparison of static and dynamic cerebral autoregulation measurements. Stroke. 1995; 26(6):1014-1019. [PubMed: 7762016]

11. Mitsis GD, et al. Nonlinear modeling of the dynamic effects of arterial pressure and $\mathrm{CO}_{2}$ variations on cerebral blood flow in healthy humans. IEEE Trans Biomed Eng. 2004; 51:1932-1943. [PubMed: 15536895]

12. Chan GS, et al. Contribution of arterial Windkessel in low-frequency cerebral hemodynamics during transient changes in blood pressure. J Physiol. 2011; 110(4):927-25.

13. Keage HA, et al. Cerebral vascular function in aging and dementia: a systematic review of Transcranial Doppler studies. Dementia and Geriatric Disorder. 2012; 2:258-279.

14. Classen JA, et al. Transfer function analysis of dynamic cerebral autoregulation: A white paper from the International Cerebral Autoregulation Research Network. J Cerebral Blood Flow \& Metab. 2015; 0(00):1-16.

15. Claasen JA, Zhang R. Cerebral Autoregulation in Alzheimer's disease. J Cereb Blood Flow Metab. 2011; 31(7):1572-1577. [PubMed: 21540872]

16. Hamner JW, Tan Can Ozan. Relative Contributions of Sympathetic, Cholinergic, and Myogenic Mechanisms to Cerebral Autoregulation. Stroke. 2014; 45(6):1771-1777. [PubMed: 24723314]

17. Lu K, Clark JW. Cerebral Autoregulation and Gas Exchange Studied Using a Human Cardiopulmonary Model. AJP-Heart. 2003; 286:584-601.

18. Glodzik L, et al. Cerebrovascular reactivity to carbon dioxide in Alzheimer's disease. A review. $\mathbf{J}$ Alz Dis. 2013; 35(3):427-440.

19. Ursino M, et al. Cerebral hemodynamics during arterial and $\mathrm{CO} 2$ pressure changes: in vivo prediction by a mathematical model. Am J Physiol Heart Circ Physiol. 2000; 279(5):H2439_ H2455. [PubMed: 11045982]

20. Ursino $\mathrm{M}$, et al. Interaction among autoregulatino, $\mathrm{CO}_{2}$ reactivity, and intracranial pressure: a mathematical model. Am J Physiol Heart Circ Physiol. 1998; 274:5.

21. Ursino $M$, et al. Intracranial pressure dynamics in patients with acute brain damage: a critical analysis with the aid of a mathematical model. IEEE Transactions on BME. 1995; 42(6):529-540.

22. Villalba N, et al. Traumatic brain injury disrupts cerebrovascular tone through endothelial inducible nitric oxide synthase expression and nitric oxide gain of function. J Am Heart Assoc. 2014; 10(1161)

23. Aaslid R, et al. Cerebral autoregulation dynamics in humans. Stroke. 1989; 20:45-52. [PubMed: 2492126]

24. Rao R. The role of Carotid Stenosis in Vascluar Cognitive Impairment. Eur Neurol. 2001; 46:6369. [PubMed: 11528153]

25. Kelleher RJ, Soiza RL. Evidence of endothelial dysfunction in the development of Alzheimer's disease: Is Alzheimer's a vascular disorder? Am J Cardiovasc Dis. 2013; 3(4):197-226. [PubMed: 24224133] 
26. Zhang R, et al. Transfer function analysis of dynamic cerebral autoregulation in humans. Am J Physiol. 1998; 274:233-241.

27. Zhang R, et al. Dynamic pressure-flow relationship of the cerebral circulation during acute increase in arterial pressure. J Physiol. 2009; 587(11):2567-77. [PubMed: 19359366]

28. Daun S, Tjardses T. Modeling, analysis, and calculation of cerebral hemodynamics. Comp \& Math Methods in Med. 2007; 8:205-223.

29. Dhoat $S$, et al. Vascular compliance is reduced in vascular dementia and not in Alzheimer's diease. Age \& Aging. 37:653.

30. Diamond SG, et al. A cerebrovascular response model for functional neuroimaging including dynamic cerebral autoregulation. Math Biosci. 2009; 220(2):102-117. [PubMed: 19442671]

31. Payne SJ, Tarassenko L. Combined Transfer Function Analysis and Modelling of Cerebral Autoregulation. Annals of Biomed Eng. 2006; 34(5):847-58.

32. Payne SJ. A model of the interaction between autoregulation and neural activation in the brain. Math Biosci. 2006; 204:260-281. [PubMed: 17010387]

33. Peng T, et al. Multivariate System Identification for Cerebral Autoregulation. Annals of Biomed Eng. 2008; 36:308-20.

34. Marmarelis VZ, et al. Time-Varying Modeling of Cerebral Hemodynamics. IEEE Trans Biomed Eng. 2014; 61(3):694-704. [PubMed: 24184697]

35. Marmarelis VZ, et al. Closed-loop analysis of cerebral flow autoregulation using Principal Dynamic Modes. Annals of Biomed Eng. 2013; 41(5):1029-1048.

36. Marmarelis VZ, et al. Linear and nonlinear modeling of cerebral flow autoregulation using Principal Dynamic Modes. Biomed Eng J. 2012; 6:42-55.

37. Marmarelis VZ. Model-based Quantification of cerebral hemodynamics as a physiomarker for Alzheimer's disease? Annals of Biomed Eng. 2013; 41:2296-2317.

38. Marmarelis VZ. Nonlinear Dynamic Modeling of Physiological Systems. Wiley-Interscience. 2004:100-4.

39. Tzeng Y, et al. Determinants of human cerebral pressure-flow velocity relationships: new insights from vascular modelling and $\mathrm{Ca}^{2+}$ channel blockade. J Physiol. 2011; 589(13):3263-3274.

[PubMed: 21540346] 


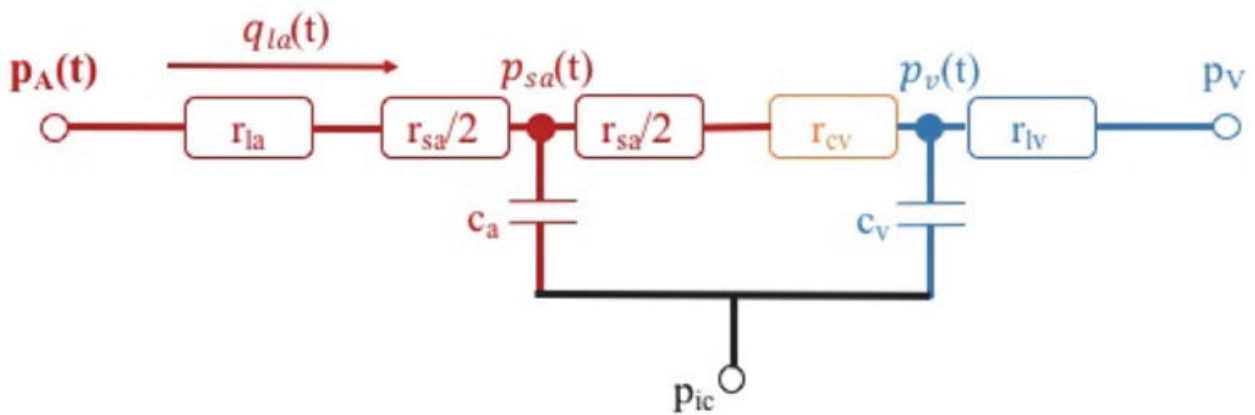

Figure 1.

Equivalent circuit representation of the $\mathrm{CH}$ model. The model is composed of three segments: 1) arterial (red), 2) capillary/venule (orange), and 3) venous (blue). 


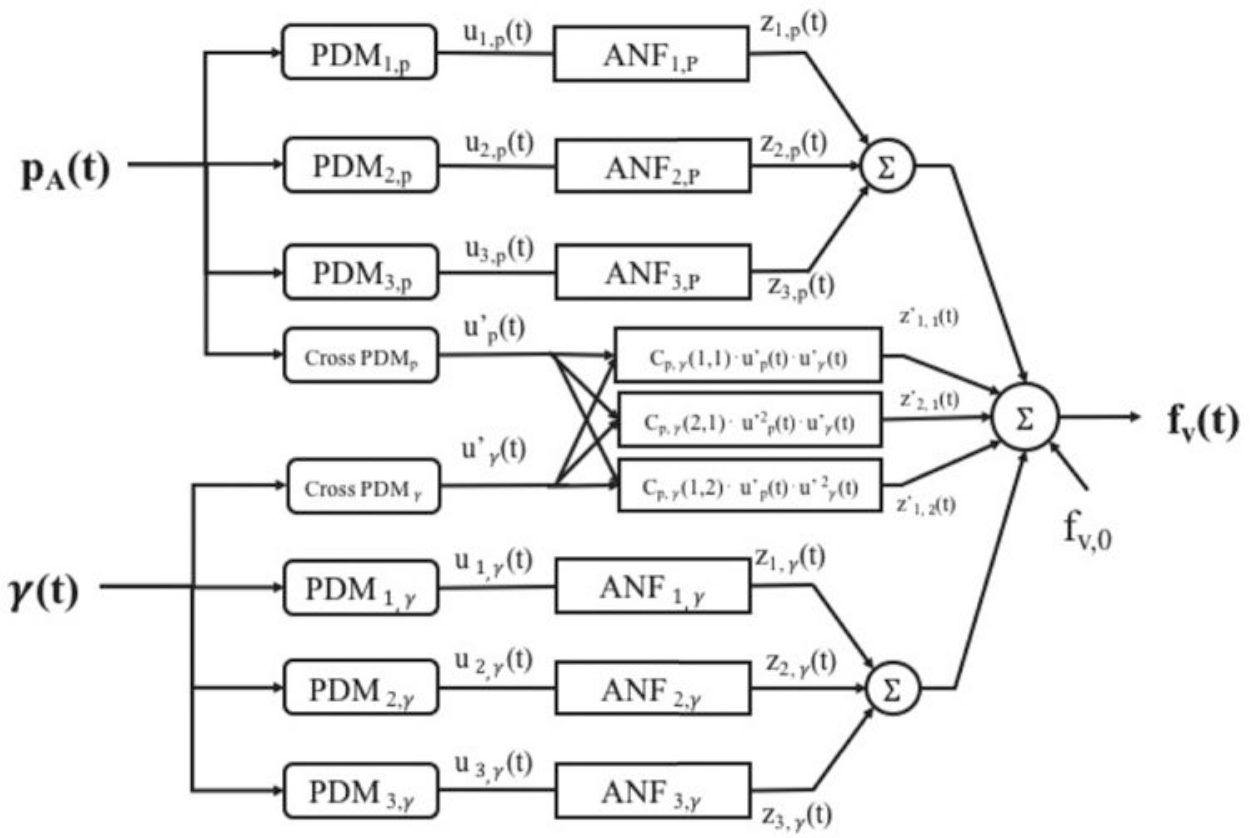

Figure 2.

The block-diagram of the PDM model. Three PDMs for the MABP input, three PDMs for the $\mathrm{ETCO}_{2}$ input, and one cross PDM for both inputs were used to approximate the $\mathrm{CH}$ model. 

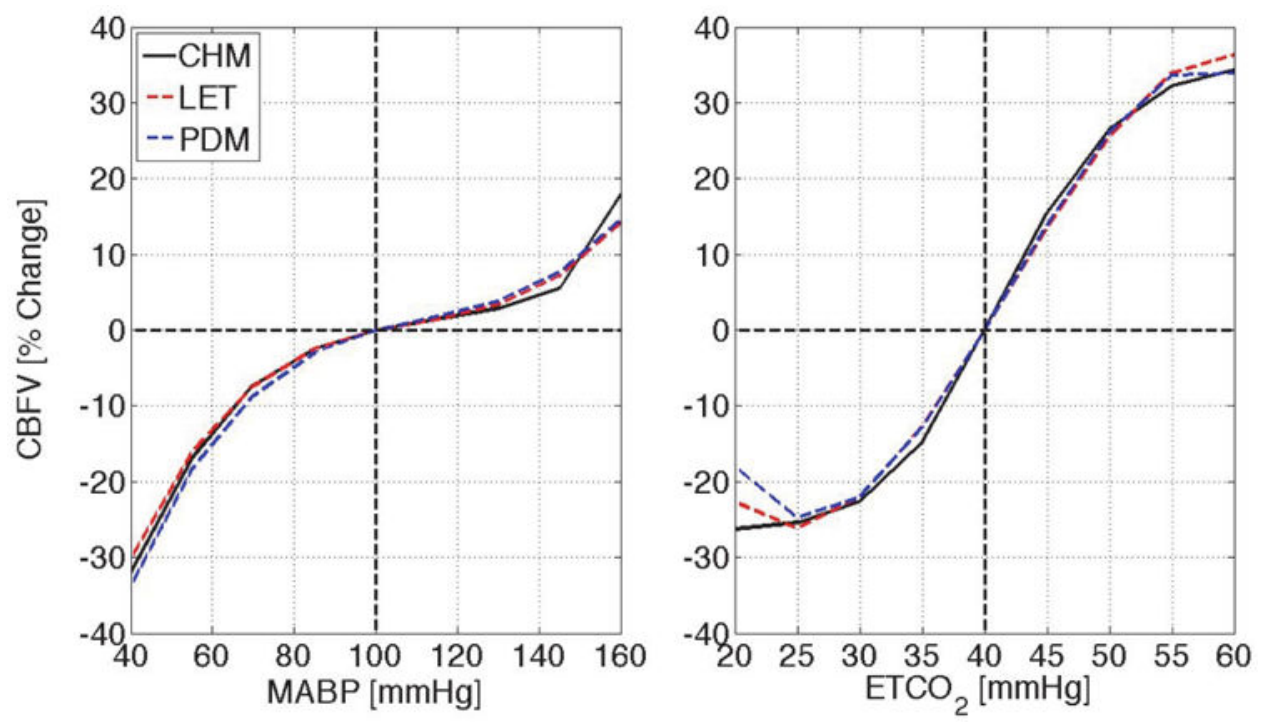

Figure 3.

The CA (left) and VR (right) curve predicted by the $\mathrm{CH}$ model (black) and the estimated curves using the LET (red) and PDM model (blue). 

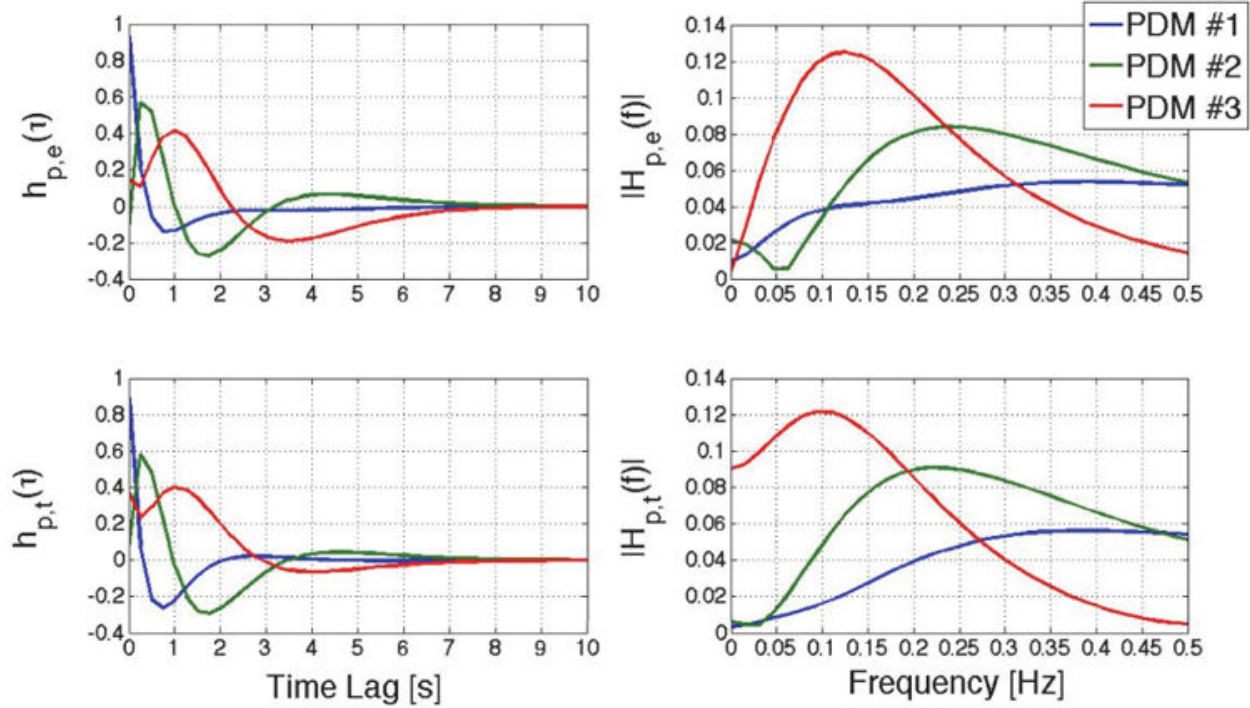

Figure 4.

Three global PDMs obtained from experimental kernels estimated from 10 healthy human MABP and CBFV measurements (top) and the PDMs obtained from the theoretical kernels estimated from 10 simulated MABP and CBFV data (bottom) in time (left) and frequency (right) domain. 

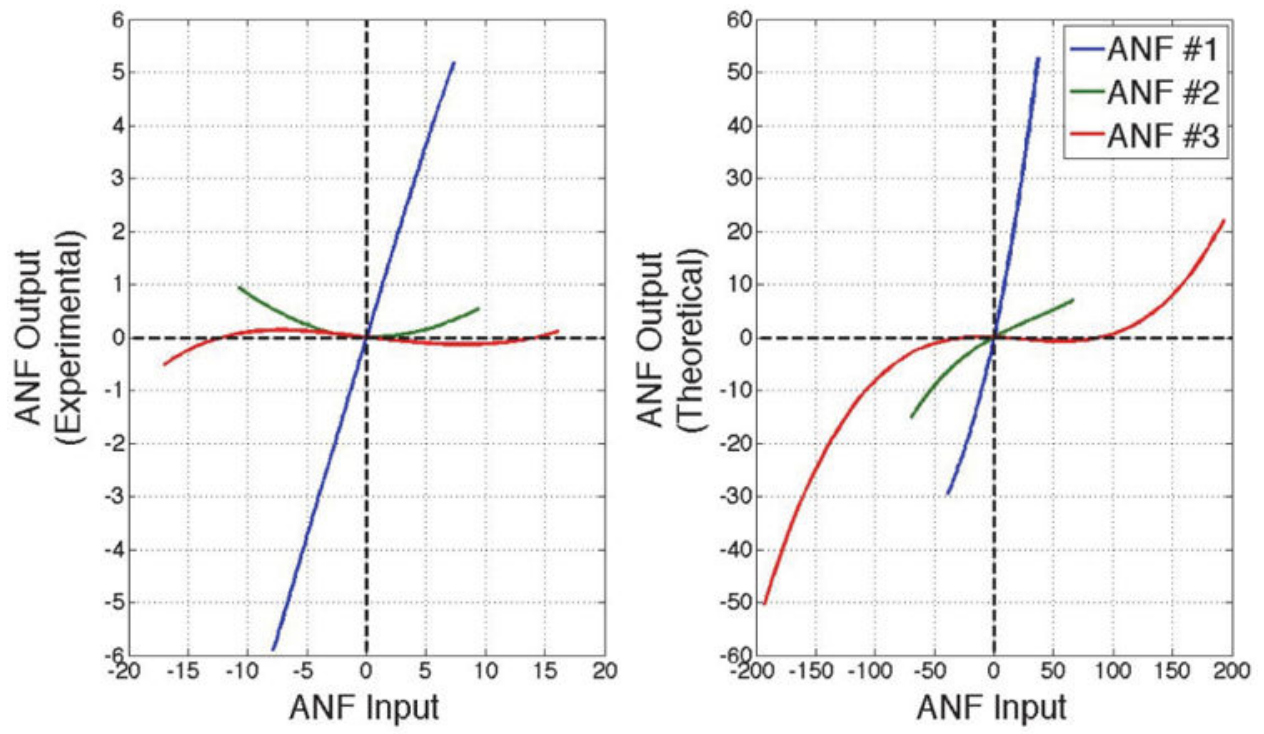

Figure 5.

The ANFs of the first (blue), and second (green), and third (red) branch of the MABP input, averaged between the 10 estimated PDM models using the experimental (left) and simulated (right) model. 

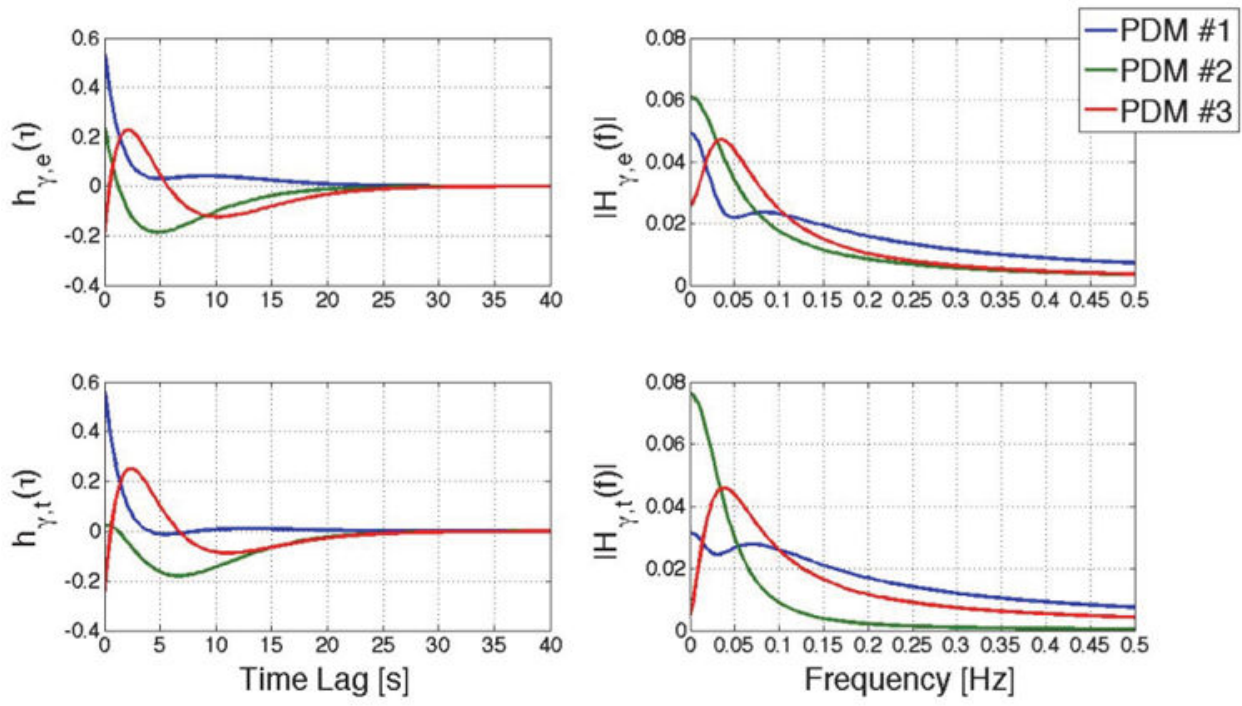

Figure 6.

Three global PDMs obtained from experimental kernels estimated from 10 healthy human $\mathrm{ETCO}_{2}$ and CBFV measurements (top) and the PDMs obtained from the theoretical kernels estimated from 10 simulated $\mathrm{ETCO}_{2}$ and $\mathrm{CBFV}$ data (bottom) in time (left) and frequency (right) domain. 

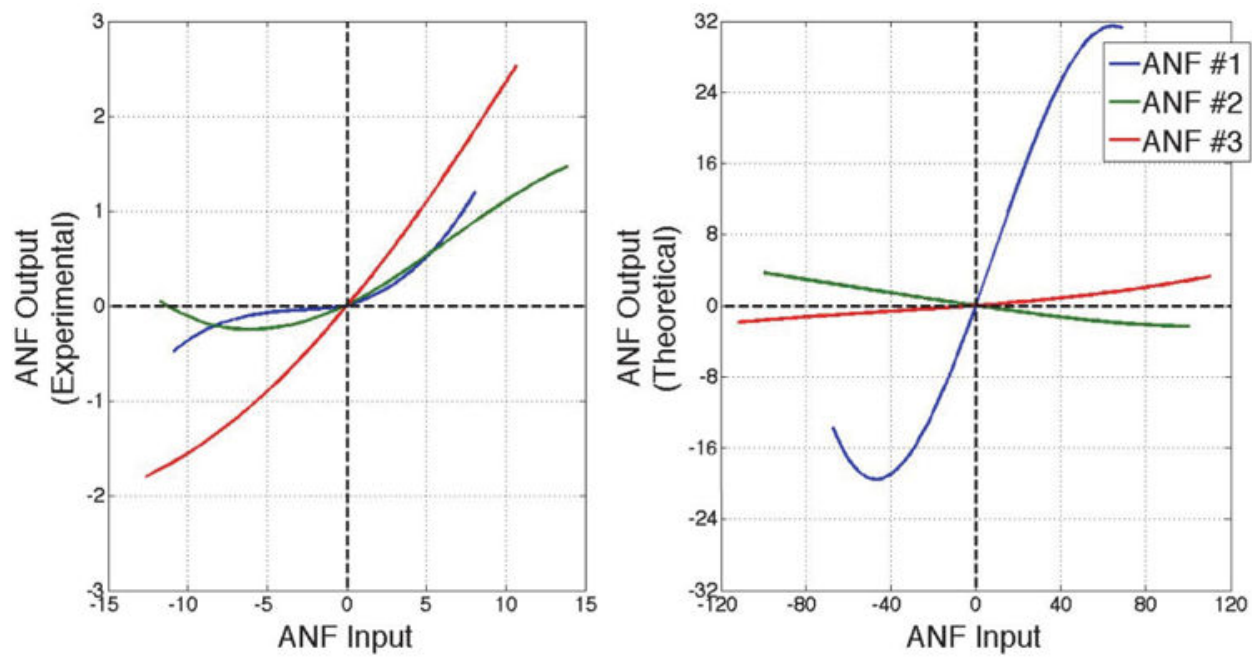

Figure 7.

The ANFs of the first (blue) and second (red) branch of the $\mathrm{ETCO}_{2}$ input, averaged between the 10 estimated PDM models using the experimental (left) and simulated (right) data. 


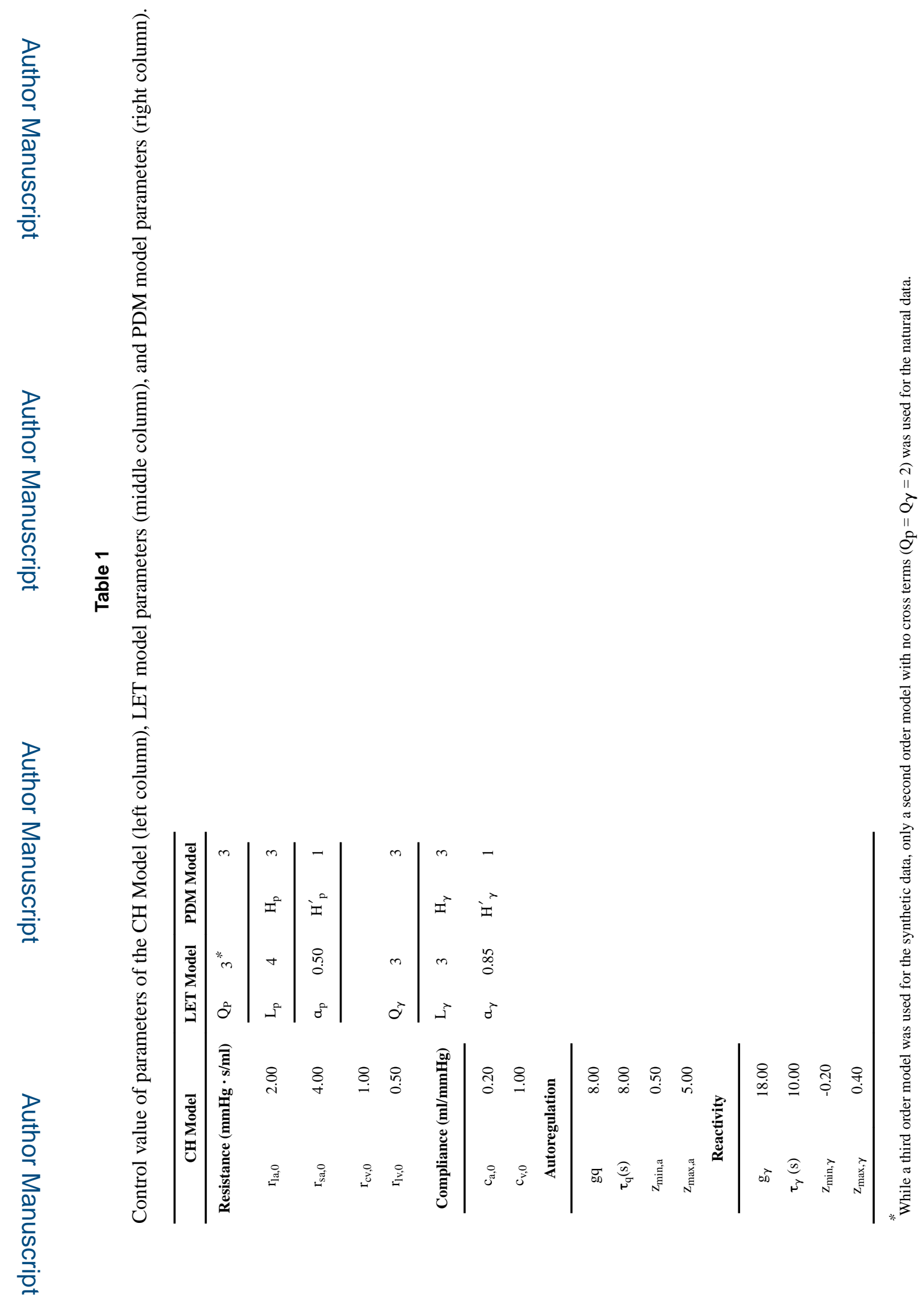

IEEE Trans Biomed Eng. Author manuscript; available in PMC 2018 May 01. 